\title{
Conducta antisocial y delictiva en adolescentes y jóvenes colombianos ${ }^{7}$
}

\author{
Ana Fernanda Uribe Rodríguez \\ PhD en Psicología Clínica y de la Salud de la \\ Universidad Pontificia Bolivariana \\ Bucaramanga, Colombia \\ Correo electrónico: anafernanda.uribe@upb.edu.co \\ Ana María Sanabria \\ Psicologa Magister Psicología Social \\ Pontificia Universidad Javeriana \\ Cali, Colombia \\ Correo electrónico: anasanabria@javerianacali.edu.co
}

\section{Linda Teresa Orcasita}

Mg. Familia

Pontificia Universidad Javeriana

Cali, Colombia

Correo electrónico: lindaorcasita@gmail.com

\section{Jennifer Castellanos Barreto \\ Psicóloga \\ Universidad Pontificia Bolivariana \\ Bucaramanga, Colombia \\ Correo electrónico: jenyfer-19@hotmail.com}

\section{Resumen}

Objetivo: descripción de conductas antisociales y delictivas en adolescentes colombianos y la diferenciación en función de variables sociodemográficas. Método: se realizó una investigación transversal con diseño descriptivo e inferencial en la que se contó con una muestra de 770 sujetos, de los cuales el $47.8 \%$ eran hombres y el $52.2 \%$ fueron mujeres, con edades comprendidas entre los $10 \mathrm{a}$ los 23 años, pertenecientes a colegios públicos de cinco ciudades de Colombia. Para la recolección de los dato se aplicó el Cuestionario de Conductas Antisociales-Delictivas- (A-D) (Seisdedos, 1995). Resultados: los adolescentes presentan mayores conductas antisociales que delictivas, y siguen presentándose con mayor frecuencia en los hombres. Con la relación a las ciudades, en Bogotá los adolescentes presentan más conductas antisociales, mientras que en Cali los adolescentes presentan mayores conductas delictivas. Conclusión: se identifica la importancia de estudios en los que se tenga en cuenta otras variables de tipo individual, grupal y social, así como el contexto familiar, los cuales podrían contribuir de manera significativa a la mejora y creación de programas de prevención en los menores.

Palabras clave adolescentes, conducta antisocial, conducta delictiva.

7 Para citar este artículo: Uribe, A.F., Sanabria, A.M., Orcasita, L., \& Castellanos, J. (2016). Conducta antisocial y delictiva en adolescentes y jóvenes colombianos. Informes Psicológicos, 16(2), pp. 103-119. http://dx.doi.org/10.18566/ infpsicv16n2a07 


\section{Anti-social and criminal behavior in Colombian adolescents and youth}

Abstract

Objective: to describe antisocial and criminal behavior in Colombian adolescents and their differentiation according to socio-demographic variables. Method: a cross-sectional research was applied with a descriptive and inferential design, which included a sample of 770 subjects, of whom $47.8 \%$ were male and $52.2 \%$ female, with ages ranging from 10 to 23 , belonging to public schools in five cities in Colombia. For the collection of data, the Antisocial-Criminal Behavior Questionnaire (A-D) was applied (Seisdedos, 1995). Results: teens have a higher anti-social criminal behavior, and this appears more frequently in men. In relation to the cities, Bogota teens present the more antisocial behavior, while in Cali teens tend to have major criminal offences. Conclusion: studies that take into account other variables of individual, group and social types, as well as the family context, become important. These could contribute significantly in the improvement and creation of prevention programs in minors.

Keywords

\section{Conduta antissocial e delitiva em adolescentes e jovens colombianos}

\section{Resumo}

Objetivo: descrição de condutas antissociais e delitivas em adolescentes colombianos e a diferenciação em função de variáveis sócio-demográficas. Método: se realizou uma investigação transversal com desenho descritivo e inferencial na que se contou com uma amostra de 770 sujeitos, dos quais $47,8 \%$ eram homens e $52,2 \%$ foram mulheres, com idades compreendidas entre os 10 aos 23 anos, pertencentes a colégios públicos de cinco cidades da Colômbia. Para a recolecção dos dado se aplicou o Questionário de Condutas Antissociais-Delitivas- (A-D) (Seisdedos, 1995). Resultados: os adolescentes apresentam maiores condutas antissociais que delitivas, e seguem apresentando com maior frequência nos homens. Com a relação às cidades, em Bogotá os adolescentes apresentam mais condutas antissociais, enquanto que em Cali os adolescentes apresentam maiores condutas delitivas. Conclusão: se identifica a importância de estudos nos que se tenha em conta outras variáveis de tipo individual, grupal e social, assim como o contexto familiar, os quais poderiam contribuir de maneira significativa à melhora e criação de programas de prevenção nos menores.

Palavras chave adolescentes, conduta antissocial, conduta delitiva. 


\section{ntroducción}

La adolescencia es una etapa que se caracteriza por el despertar de la curiosidad y de la experimentación, así mismo, es un periodo de transformación continua, la cual requiere ajustes a cambios biológicos, emocionales y sociales del propio desarrollo. Al ser un periodo crítico que enfrenta la persona, aparece también el incremento de problemas del comportamiento, particularmente antisociales que en la mayoría de las veces, cuando son con frecuencia y desde temprana edad, con el tiempo trascienden a delictivo (Gaeta \& Galvanovski, 2011; Sanabria \& Uribe, 2009). Así mismo, la adolescencia es concebida como una etapa de transición muy significativa para el desarrollo de la personalidad, ya que se caracteriza por el desprendimiento del mundo infantil a la búsqueda de un mundo adulto (Cabrera, González, Vargas, \& Franco, 2012).

En la actualidad, la delincuencia juvenil se ha configurado como un problema que cada vez pone en alerta a la población mundial (Herrera \& Morales, 2005; Montero, 2014), ya que al tratarse de una forma de violencia visible, que en algunas ocasiones se configura en pandillas escolares (Sanabria \& Uribe, 2010), ha traído consigo un aumento en los índices de homicidios, lesiones y discapacidad en adolescentes y jóvenes (Cabrera et al., 2012; OMS, 2003). Adicionalmente, la delincuencia "implica altos costos económicos y sociales por la desintegración de las familias, la pérdida de vidas, la atención de la salud, así como programas educativos y de rehabilitación" (Gaeta \& Galvanovski, 2011, p. 48). Por lo tanto, la participación de los adolescentes y jóvenes en actos antisociales y delincuenciales repercute de una forma significativa en el desarrollo personal, social y económico de un país (OMS, 2003).

Se dice que los adolescentes, quienes a muy temprana edad presentan conductas antisociales y delictivas durante un largo tiempo, se encuentran en alto riesgo de seguir presentado estas mismas conductas en una edad más adulta (Giraldo, Ocampo, Tuta, Gallego, \& López, 2015; Pérez, Molero, Gázquez, \& Abad, 2014); lo que significaría que en su juventud y/o adultez, éstos presenten problemas asociados a alto riesgo como consumo de sustancias psicoactivas, dificultades académicas, conductas sexuales de riesgo entre otras (Sanabria \& Uribe, 2009; Sanabria \& Uribe, 2010). De acuerdo con Duque, Klevens y Montoya (2007, como se citó en Cabrera et al., 2012) esta etapa inicia antes de los 12 años, en donde presentan comportamientos de oposición o terquedad; a medida del crecimiento de los niños, se presentan también comportamientos desafiantes y de desobediencia, que en ocasiones los lleva a evadir la autoridad.

Investigaciones señalan que los comportamientos antisociales comienzan desde edades comprendidas entre los 12 a 14 años, partiendo desde agresiones, falta de respeto hacia la autoridad, quebrantamiento de la norma, entre otras, que son conductas socialmente no aceptadas (Cabrera et al., 2012; Pelegrín \& Garcés de Los Fayos, 2009; Sanabria \& Uribe, 2009; Sanabria \& Uribe, 2010). 
Para Hibbs y Jensen (1996) una conducta antisocial es "considerada como la oposición a las reglas de la sociedad" (como se citó en Gaeta \& Galvanovski, 2011, p. 48). En un sentido más amplio, Garaigordobil (2005) la define como "cualquier conducta que refleje infringir reglas sociales y/o sea una acción contra los demás. En concreto, se exploran conductas antisociales asociadas al gamberrismo y a conductas de trasgresión de normas sociales en relación con la edad" alguna de ellas como "romper objetos, agredir a personas, falsificar notas, no asistir al colegio o llegar tarde intencionalmente, copiar en un examen, robar, ensuciar las calles y las aceras rompiendo botellas o vertiendo las basuras, tirar piedras a la gente, tirar piedras a casas, coches o trenes..." (p.198).

Por otra parte, la conducta delictiva es definida como la "designación legal, basada generalmente en el contacto con las leyes de justicia del país en que se encuentra el niño o adolescente" (Kazdin \& Buela-Casal, 1996, p. 31). Es decir, que la delincuencia son desviaciones de la conducta que "comprenden actos legalmente definidos como delictivos, tales como violencia, robo o vandalismo, hasta una variedad de conductas no delictuosas, como agresión, peleas, prepotencia y, en general, un quebrantamiento serio de las normas en el hogar y en la escuela" (Kazdin, 1988; Erickson, 1992, como se citó en Gaeta \& Galvanovski, 2011, p.48). En este sentido, es importante mencionar que la conducta delictiva no es un constructo psicológico, sino una categoría jurídico-legal, en la que no se agrupan todos los delincuentes, sino que comparten la misma conducta de delinquir, en donde este tipo de conducta reúne variables psicológicas consistentes que se configuran en un patrón conductual al cual se le denomina "comportamiento antisocial"

Por ende, es requerido señalar que el comportamiento antisocial y el delito no son bidireccionales, es decir, todo delito constituye un tipo de comportamiento antisocial, pero no todo comportamiento antisocial es un delito, en tanto no haya infringido la ley de una Nación (Cabrera et al., 2012; Morales, 2008). De este modo, las conductas antisociales tienen consecuencias para los adolescentes, como procesos educativos sin éxito (Cabrera et al., 2012), expulsión e incluso hospitalización, algunas veces en su relación con el otro dejan víctimas y en ocasiones al llegar a la edad adulta corren el riesgo de versen involucrados en problemas de alto riesgo psicosocial (Sanabria \& Uribe, 2009; Sanabria \& Uribe, 2010).

Por otro lado, las investigaciones han señalado que existen diferencias en la manifestación de la conducta antisocial en función del género (Pérez et al., 2014); los hombres presentan más conductas antisociales que las mujeres, aunque las diferencias no son significativas (Garaigordobil, 2005). Diferentes países muestran la participación de adolescentes hombres en distintos hechos antisociales y delictivos en muchas más ocasiones que las mujeres, especialmente cuando el ambiente y las circunstancias lo favorecen (Gaeta \& Galvanovskis, 2011; Herrera \& Morales, 2005; Montero, 2014; Pérez et al., 2014; Sanabria \& Uribe, 2009; Uribe, 2005). Igualmente, los hombres presentan más conductas agresivas que ellas, 
mientras que en la mujer las conductas se encuentran orientadas a hacer trampas (en examen o información de resultados) y llegar tarde al colegio, lo cual concluye que las mujeres tienden a involucrarse en más comportamientos antisociales no agresivos en comparación con los hombres (Gaeta \& Galvanovskis, 2011).

En este mismo sentido, el comportamiento antisocial y delictivo tiene un inicio temprano en los jóvenes, y las conductas violentas tiende a aumentar con la edad (Gaeta \& Galvanovskis, 201; Kazdin, 1995), algunos adolescentes presentan sus conductas antisociales desde los 12 años, la cual aumenta progresivamente con la edad (Gaeta \& Galvanovskis, 2011; Sanabria \& Uribe, 2009). En los resultados de Gaeta \& Galvanovskis (2011), se evidencia que los jóvenes entre 18 y 20 años de edad son más propensos a realizar conductas antisociales y delictivas, en comparación con los de 14 años y menos. Entre los adolescentes de 12 a 14 y 15 a 17 años hay diferencias significativas en la propensión a conductas antisociales, pero no propiamente a conductas delictivas, estos resultados son equiparables a los hallados en las investigaciones de Uribe (2005) y Sanabria y Uribe (2009, 2010) en adolescentes colombianos. Así mismo, en otro estudio realizada por Garaigordobil (2005) confina que aquellos adolecentes que presenta conductas antisociales mantienen una relación inversamente proporcional con conductas positivas como, autocontrol, liderazgo, prosociales, asertivas, de adaptación social, así como autoconcepto y con empatía; por ende, los adolescentes quienes presentan este tipo de conductas tienen bajo auto-concepto, autoestima, poca empatía y alta impulsividad (Arce, Fariña \& Vázquez, 2011).

De acuerdo con lo anterior, es relevante señalar que la adolescencia es un periodo de mayor autonomía, en donde la autonomia y la delincuencia son directamente proporcionales; es en este período de transición, en donde el rol del control de los padres se vuelve en ocasiones confuso, ya que el control puede influir de forma negativa o positiva en las conductas del adolescente (Hoeve et al., 2009). En este caso, es relevante la asociación con los estilos de crianza, de los que se dice que la paternidad temprana complaciente se halla positivamente relacionados con el comportamiento criminal en los adultos (Schroeder, Bulanda, Giordana, \& Cernkovich, 2010). De este modo, en un estudio longitudinal con adolescentes en Estado Unidos, se comprobó que la falta de control de los padres se encuentra asociado con un mayor nivel de delincuencia en la adolescencia y también con el comportamiento criminal en la adultez temprana (Harris-McKoy, \& Cui, 2013). Así mismo, en una revisión sistemática, en donde se tuvo en cuenta la relación de los padres con los adolescentes, se confirmó la importancia de las prácticas de crianza y su influencia sobre el comportamiento antisocial de los adolescentes, ya que los niveles de unión entre padres y adolescentes, el bajo control de los padres, baja supervisión y baja orientación favorece la conducta antisocial de los adolescentes e incluso en la edad adulta joven. Por lo tanto, la crianza positiva y consistente, la participación de los padres y los buenos modelos de conducta de los padres, son factores orientados a reducir el comportamiento antisocial de los 
adolescente (Human-Hendricks \& Roman, 2014)

Finalmente, este tipo de conductas también han sido relacionadas con el tipo de personalidad (Morán, Carmona, \& Fínez, 2016). En los adolescentes se confirma la existencia de relaciones significativas entre determinados rasgos de personalidad (psicoticismo, Impulsividad, falta de autocontrol, despreocupación, atrevimiento) y la conducta antisocial y delictiva (López \& López, 2003). Por tanto, la predicción de rasgo muy estable de la personalidad, también permite predecir de forma independiente futuras conductas antisociales desde edades muy tempranas, teniendo en cuenta que la mayoría de exposición a factores de riesgo se encuentra en la adolescencia. Por tanto, parece que la psicopatía es un rasgo en especial relevante, pues se distingue claramente del comportamiento antisocial en general e influye de manera diferente, en tiempo y forma, al sujeto (Jordá \& Besteiro, 2013). Adicionalmente, se deben tener en cuenta que en la formación de la personalidad, intervienen otros factores de tipo externo que se encuentran el exosistema en donde se desarrolla el adolescente, como el ambiente desorganizado del barrio, las ventas de drogas y alcohol, éstas son variables ambientales que también se deben tener en cuenta en la predicción delincuencial (Giraldo et al., 2015; Peña, 2010; Sanabria \& Uribe, 2010).

Con base en lo anterior, se establece como objetivo central la descripción de conductas antisociales y delictivas en adolescentes colombianos y la diferenciación en función de variables sociodemográficas.

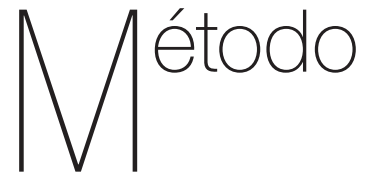

\section{Diseño}

Se realizó una investigación no experimental transversal con diseño descriptivo e inferencial, con el propósito de indagar la incidencia de las variables en la población (Hernández, Fernández, \& Baptista, 2010)

\section{Participantes}

La muestra estuvo conformada por 770 sujetos, de los cuales el $47.8 \%$ eran hombres y el $52,2 \%$ fueron mujeres, con edades comprendidas entre los 10 a los 23 años. Los adolescentes se encontraban cursando bachillerato, es decir de grado sexto a once, pertenecientes a colegios públicos de cinco ciudades de Colombia. El mayor porcentaje (49.4\%) de los adolescentes era de estrato socio económico dos.

\section{Instrumento}

Cuestionario de Conductas Antisociales-Delictivas- $(A-D)$, elaborado por Seisdedos (1995), el cual incluye dos subescalas la conducta antisocial (20 ítems) y la conducta delictiva (20 ítems). El tipo de respuesta es dicotómica "sí" o "no" frente a comportamientos relacionados con los comportamientos desviados. La adaptación colombiana fue realizada por Uribe, Bermúdez y BuelaCasal (2005, citado por Uribe, 2005). El cuestionario cuenta con un análisis 
factorial que confirma la estructura de la escala y un análisis de confiabilidad con un alfa de Cronbach .90 para el instrumento en general.

\section{Procedimiento}

Una vez obtenido el permiso de cada una de las instituciones, y así mismo obtener el asentimiento informado de los padres de familia para los menores de 16 años y el consentimiento informado de los participantes mayores de 16 años, que cumplían los criterios de selección, se procedió a la aplicación de los instrumentos en cada institución. Cabe aclarar que previamente se les informó a los participantes que su inclusión en el estudio era completamente voluntaria, anónima (no debían escribir su nombre en los instrumentos) y que no obtendrían incentivos económicos por su participación. Una vez recopilados los datos fue creada la base de datos utilizando el paquete estadístico Statistical Package for Social Science (SPSS) y finalmente se realizaron los respectivos análisis.

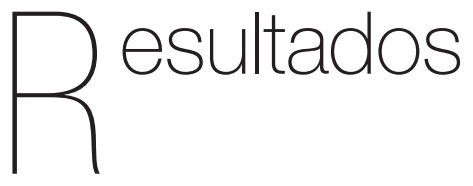

En este apartado se presentan, en primera instancia, los resultados de la conducta antisocial y la conducta delictiva en adolescentes colombianos. Posteriormente, los resultados de la conducta antisocial y delictiva en función del sexo, ciudad, escolaridad y estrato (Tabla 1).
Tabla 1.

Distribución de la Muestra en función de la ciudad, edad, sexo y escolaridad

\begin{tabular}{|c|c|c|c|}
\hline Variables & & Frecuencia & $\%$ \\
\hline \multirow{5}{*}{ Ciudad } & Bogotá & 103 & 12.8 \\
\hline & Pasto & 164 & 20.4 \\
\hline & Manizales & 132 & 16.4 \\
\hline & Armenia & 279 & 34.7 \\
\hline & Cali & 127 & 15.8 \\
\hline \multirow{2}{*}{ Sexo } & Hombre & 368 & 47.8 \\
\hline & Mujer & 402 & 52.2 \\
\hline \multirow{5}{*}{ Estrato } & 1 & 287 & 36.0 \\
\hline & 2 & 394 & 49.4 \\
\hline & 3 & 102 & 12.8 \\
\hline & 4 & 12 & 1.5 \\
\hline & 5 & 3 & .4 \\
\hline \multirow{6}{*}{ Escolaridad } & Sexto & 125 & 15.5 \\
\hline & Séptimo & 141 & 17.5 \\
\hline & Octavo & 147 & 18.3 \\
\hline & Noveno & 118 & 14.7 \\
\hline & Decimo & 208 & 25.9 \\
\hline & Once & 65 & 8.1 \\
\hline \multirow{4}{*}{ Edad } & 10-12 años & 153 & 19.1 \\
\hline & 13-15 años & 392 & 48.9 \\
\hline & 16-18 años & 249 & 31.1 \\
\hline & 19-23 años & 8 & .9 \\
\hline
\end{tabular}

\section{Conducta antisocial y delictiva en adolescentes colombianos}

Para la presentación de los resultados de la conducta antisocial y delictiva se realizan dos tipos de análisis, uno descriptivo y otro univariado (ANOVA), en adolescentes colombianos. Se realiza una descripción de cada una de las 
escalas del Cuestionario de Conductas Antisociales y Delictivas AD (Seisdedos, 1995). Se calcularon las puntuaciones mínimas y máximas de cada una de las escalas, su media y su desviación típica (Tabla 2).

Tabla 2

Puntuaciones mínimas, máximas, medias y desviaciones típicas en las conductas antisocial y delictiva en adolescentes colombianos

\begin{tabular}{ccccc}
\hline Escala & Mínimo & Máxima & Media & Desviación típica \\
\hline Antisocial & 0 & 20 & 5.487 & 5.457 \\
Delictiva & 0 & 20 & 1.752 & 3.447 \\
\hline
\end{tabular}

Las conductas antisociales que se presentan con mayor frecuencia en los adolescentes, son aquellas que se relacionan con el mal uso de palabras y/o groserías, aspectos relacionados al incumplimiento o llegada tarde al colegio, ingerir alimentos en lugares donde es prohibido, llamar a la puerta de alguien y salir corriendo, pelearse con otros, hacer trampa, y tirar basura al suelo así haya una papelera cerca. Estas conductas se identifican como principales, ya que más del $30 \%$ de la muestra las ha realizado (Ver Tabla 3).

Tabla 3

Descripción de frecuencia de conductas antisociales entre adolescentes colombianos

\begin{tabular}{|c|c|c|c|c|}
\hline \multirow{3}{*}{ Ítem } & \multicolumn{4}{|c|}{ Adolescentes Escolarizados } \\
\hline & \multicolumn{2}{|c|}{$\mathrm{Si}$} & \multicolumn{2}{|c|}{ No } \\
\hline & $\mathrm{F}$ & $\%$ & $\mathrm{~F}$ & $\%$ \\
\hline Alborotar o silbar en una reunión, lugar público o de trabajo. & 160 & 19.9 & 645 & 80,1 \\
\hline Salir sin permiso (del trabajo, de casa o del colegio). & 228 & 28.3 & 577 & 71.7 \\
\hline Entrar en un sitio prohibido (jardín privado, casa vacía). & 177 & 22.0 & 628 & 78.0 \\
\hline Ensuciar las calles /aceras rompiendo botellas o volcando cubos de basura. & 150 & 18.6 & 655 & 81.4 \\
\hline Decir groserías o palabras fuertes. & 324 & 40.2 & 481 & 59.8 \\
\hline Molestar a personas desconocidas o hacer daños en lugares públicos. & 127 & 15.8 & 678 & 84.2 \\
\hline Llegar tarde al trabajo, colegio o reunión. & 300 & 37.3 & 505 & 62.7 \\
\hline Hacer trampas (en examen, competición importante, información de resultados). & 295 & 36.6 & 510 & 63.4 \\
\hline Tirar basuras al suelo (cuando hay cerca una papelera o cubo). & 248 & 30.8 & 557 & 69.2 \\
\hline Hacer pintadas en lugares prohibidos (pared, encerado, mesa, etc). & 178 & 22.1 & 627 & 77.9 \\
\hline Coger fruta en un jardín /huerto que pertenece a otra persona. & 233 & 28.9 & 572 & 71.1 \\
\hline Romper o tirar al suelo cosas que son de otra persona. & 107 & 13.3 & 698 & 86.7 \\
\hline $\begin{array}{l}\text { Gastar bromas pesadas a la gente, como empujarlas den- } \\
\text { tro de un charco o quitarles la silla cuando van a sentarse. }\end{array}$ & 236 & 29.3 & 569 & 70.7 \\
\hline Llegar, a propósito, más tarde de lo permitido (a casa, trabajo, obligación). & 188 & 23.4 & 617 & 76.6 \\
\hline
\end{tabular}




\begin{tabular}{|c|c|c|c|c|}
\hline \multirow{3}{*}{ Ítem } & \multicolumn{4}{|c|}{ Adolescentes Escolarizados } \\
\hline & \multicolumn{2}{|c|}{ Si } & \multicolumn{2}{|c|}{ No } \\
\hline & $\mathrm{F}$ & $\%$ & $\mathrm{~F}$ & $\%$ \\
\hline Arrancar o pisotear flores o plantas en un parque o jardín. & 168 & 20.9 & 637 & 79.1 \\
\hline Llamar a la puerta de alguien y salir corriendo. & 295 & 36.6 & 510 & 63.4 \\
\hline Comer, cuando está prohibido, en el trabajo, clase, cine, etc). & 314 & 39.0 & 491 & 61.0 \\
\hline Contestar mal a un superior o autoridad (trabajo o calle). & 181 & 22.5 & 624 & 77.5 \\
\hline Negarse a hacer las tareas encomendadas (trabajo, clase 0 casa). & 223 & 27.7 & 582 & 72.3 \\
\hline Pelearse con otros (con golpes, insultos o palabras ofensivas) & 285 & 35.4 & 520 & 64.6 \\
\hline
\end{tabular}

En cuanto a las conductas delictivas que se presentan con mayor frecuencia en los adolescentes se encuentra el pertenecer a una pandilla que arma desorden, se mete en peleas y genera disturbios, llevar consigo un arma por si es necesaria una pelea, gastar frecuentemente en el juego más dinero del que se puede, y entrar a un club prohibido o comprar bebidas prohibidas. Estas conductas se identifican como principales, ya que más del $11.8 \%$ de la muestra las ha realizado (Ver Tabla 4).

Tabla 4

Descripción de frecuencias de conductas delictivas entre adolescentes colombianos

\begin{tabular}{|c|c|c|c|c|}
\hline \multirow{3}{*}{ Ítem } & \multicolumn{4}{|c|}{ Adolescentes Colombianos } \\
\hline & \multicolumn{2}{|c|}{$\mathrm{Si}$} & \multicolumn{2}{|c|}{ No } \\
\hline & $\mathrm{F}$ & $\%$ & $\mathrm{~F}$ & $\%$ \\
\hline Pertenecer a una pandilla que arma desorden, se mete en peleas o crea disturbios & 95 & 11.8 & 710 & 88.2 \\
\hline $\begin{array}{l}\text { Coger el carro o la moto de un desconocido para dar un pa- } \\
\text { seo, con la única intención de divertirse. }\end{array}$ & 66 & 8.2 & 739 & 91.8 \\
\hline Forzar la entrada de un almacén, garaje, guardamuebles o quiosco. & 64 & 8.0 & 741 & 92.0 \\
\hline Entrar en una tienda que está cerrada, robar o no algo. & 59 & 7.3 & 746 & 92.7 \\
\hline Robar cosas de los carros. & 55 & 6.8 & 750 & 93.2 \\
\hline Llevar algún arma (cuchillo/navaja) por si es necesaria en una pelea. & 122 & 15.2 & 683 & 84.8 \\
\hline $\begin{array}{l}\text { Planear de antemano entrar en un casa/chalet/etc. para ro- } \\
\text { bar cosas de valor (y hacerlo si se puede). }\end{array}$ & 70 & 8.7 & 735 & 91.3 \\
\hline Coger la bicicleta de un desconocido y quedarse con ella. & 31 & 3.9 & 774 & 96.1 \\
\hline Forcejear o pelear para escapar de un policía. & 74 & 9.2 & 731 & 90.8 \\
\hline Robar cosas de un lugar público (trabajo/colegio) por valor de más de $\$ 10.000$. & 54 & 6.7 & 751 & 93.3 \\
\hline Robar cosas de grandes almacenes, supermercados, etc., estando abiertos. & 63 & 7.8 & 742 & 92.2 \\
\hline Entrar en una casa/piso/etc. y robar algo (sin haberlo planeado antes). & 57 & 7.1 & 748 & 92.9 \\
\hline Robar materiales o herramientas a gente que está trabajando. & 59 & 7.3 & 746 & 92.7 \\
\hline
\end{tabular}




\begin{tabular}{|c|c|c|c|c|}
\hline \multirow{3}{*}{ Ítem } & \multicolumn{4}{|c|}{ Adolescentes Colombianos } \\
\hline & \multicolumn{2}{|c|}{$\mathrm{Si}$} & \multicolumn{2}{|c|}{ No } \\
\hline & $\mathrm{F}$ & $\%$ & $\mathrm{~F}$ & $\%$ \\
\hline Gastar frecuentemente en el juego más dinero del que se puede. & 117 & 14.5 & 688 & 85.5 \\
\hline Robar cosas o dinero en las máquinas tragamonedas, teléfono público, etc.). & 78 & 9.7 & 727 & 90.3 \\
\hline Robar ropa de un tendero o cosas de los bolsillos de ropa colgada en un gancho. & 51 & 6.3 & 754 & 93.7 \\
\hline Conseguir dinero amenazando a personas más débiles. & 56 & 7.0 & 749 & 93.0 \\
\hline Tomar drogas. & 57 & 7.1 & 748 & 92.9 \\
\hline Destrozar o dañar cosas en lugares públicos. & 77 & 9.6 & 728 & 90.4 \\
\hline Entrar en un club prohibido o comprar bebidas prohibidas. & 106 & 13.2 & 699 & 86.8 \\
\hline
\end{tabular}

\section{Resultados de la conducta antisocial y delictiva en función de variables sociodemograficas (ANOVA)}

El análisis realizado indica diferencias significativas en función del sexo en la conducta delictiva $F(1.768)=19.049$ MSE=11.716; $p<.000$ (Ver Tabla 6). Los varones adolescentes presentan una media mayor en la conducta delictiva comparada con las mujeres (Ver Tabla 5).

Tabla 5.

Medias y desviaciones típicas de la conducta antisocial y delictiva de los adolecentes en función del sexo.

\begin{tabular}{ccccc}
\hline & \multicolumn{4}{c}{ Sexo } \\
\cline { 2 - 5 } Escalas & \multicolumn{2}{c}{ Varones } & \multicolumn{2}{c}{ Mujeres } \\
\cline { 2 - 5 } M & M & D.T & M & D.T \\
\cline { 2 - 5 } Antisocial & 5.75 & 5.75 & 5.16 & 4.98 \\
Delictiva & 2.33 & 3.83 & 1.25 & 2.99 \\
\hline
\end{tabular}

Tabla 6.

Grados de libertad, media cuadrática, Fy nivel de significancia de la conducta antisocial y delictiva de los adolescentes en función del sexo.

\begin{tabular}{ccccc}
\hline Escalas & gl & $\begin{array}{c}\text { Media } \\
\text { cuadrática }\end{array}$ & F & Sig \\
\hline Antisocial & 1 & 67.730 & 2.351 & .126 \\
Delictiva & 1 & 223.178 & 19.049 & .000 \\
\hline
\end{tabular}

El análisis realizado indica diferencias significativas en función de la ciudad en la conducta antisocial $F(4.800)=M S E=28.578 ; \quad p<.000$ y en la conducta delictiva $\mathrm{F}(4.800)=6.518$; MSE=11.565; $\quad p<.000$ (Ver Tabla 7 y 8). En el análisis post-hoc mediante la prueba DSM se encontraron diferencias significativas mínimas en la conducta antisocial entre los adolescentes de Bogotá con respecto a los de Pasto, Manizales, Armenia y Cali. Así mismo se muestra el carácter progresivo de las dos conductas (Ver tabla 7). Respecto a la conducta delictiva, se encontraron diferencias significativas entre los adolescentes Armenia y Cali, estos últimos con la mayor puntuación (Ver Tablas 7 y 8). 
Tabla 7

Medias y desviaciones típicas de la conducta antisocial y delictiva de los adolecentes en función de la Ciudad.

\begin{tabular}{|c|c|c|c|c|c|c|c|c|c|c|}
\hline \multirow{3}{*}{ Escala } & \multicolumn{8}{|c|}{ Ciudad } & & \\
\hline & \multicolumn{2}{|c|}{ Bogotá } & \multicolumn{2}{|c|}{ Pasto } & \multicolumn{2}{|c|}{ Manizales } & \multicolumn{2}{|c|}{ Armenia } & \multicolumn{2}{|c|}{ Cali } \\
\hline & M & D.T & M & D.T & M & D.T & $M$ & D.T & M & D.T \\
\hline Antisocial & 7.92 & 5.02 & 4.48 & 5.06 & 4.57 & 4.87 & 6.03 & 5.81 & 4.53 & 5.34 \\
\hline Delictiva & 1.38 & 2.79 & 1.15 & 2.75 & .95 & 2.27 & 2.31 & 3.75 & 2.42 & 4.55 \\
\hline
\end{tabular}

Tabla 8

Grados de libertad, media cuadrática, F y nivel de significancia de la conducta antisocial y delictiva de los adolescentes en función de la ciudad.

\begin{tabular}{ccccc}
\hline Escalas & GI & $\begin{array}{c}\text { Media } \\
\text { cuadrática }\end{array}$ & F & Sig \\
\hline Antisocial & 4 & 271.090 & 9.486 & .000 \\
Delictiva & 4 & 75.378 & 6.518 & .000 \\
\hline
\end{tabular}

Tabla 9

Medias y desviaciones típicas de la conducta antisocial y delictiva de los adolecentes en función del estrato.

\begin{tabular}{|c|c|c|c|c|c|c|c|c|c|c|}
\hline \multirow{3}{*}{ Escala } & \multicolumn{10}{|c|}{ Estrato } \\
\hline & \multicolumn{2}{|c|}{1} & \multicolumn{2}{|c|}{2} & \multicolumn{2}{|c|}{3} & \multicolumn{2}{|c|}{4} & \multicolumn{2}{|c|}{5} \\
\hline & $M$ & D.T & $M$ & D.T & $M$ & D.T & M & D.T & $M$ & D.T \\
\hline Antisocial & 4.68 & 5.26 & 5.93 & 5.48 & 6.31 & 5.62 & 4.58 & 6.34 & 4.66 & 4.50 \\
\hline Delictiva & 1.43 & 2.98 & 1.80 & 3.57 & 2.40 & 4.04 & 2.58 & 3.77 & 3.33 & 5.77 \\
\hline
\end{tabular}

Tabla 10

Grados de libertad, media cuadrática, fy nivel de significancia de la conducta antisocial y delictiva de los adolescentes en función del estrato.

\begin{tabular}{ccccc}
\hline Escalas & Gl & $\begin{array}{c}\text { Media } \\
\text { cuadrática }\end{array}$ & F & Sig \\
\hline Antisocial & 4 & 86.584 & 2.928 & .020 \\
\hline Delictiva & 4 & 29.568 & 1.873 & .113 \\
\hline
\end{tabular}

El análisis realizado indica diferencias significativas en función de la
El análisis realizado indica diferencias significativas en función del estrato en la conducta antisocial $F(4.793)=2.928$; MSE=29.568 p<.020 (Ver Tablas 9 y 10). En el análisis post-hoc mediante la prueba DSM se encontraron diferencias significativas mínimas en la conducta antisocial presentándose mayor puntuación en los adolescentes de estrato 2 y 3 , sin embargo, en cuanto a la conducta delictiva se identifica mayor puntuación en el estrado 4 y 5 . 
Tabla 11

Medias y desviaciones típicas de la conducta antisocial y delictiva de los adolescentes en función de la escolaridad

\begin{tabular}{|c|c|c|c|c|c|c|c|c|c|c|c|c|}
\hline \multirow{2}{*}{ Escala } & \multicolumn{2}{|c|}{ Sexto } & \multicolumn{2}{|c|}{ Séptimo } & \multicolumn{2}{|c|}{ Octavo } & \multicolumn{2}{|c|}{ Noveno } & \multicolumn{2}{|c|}{ Decimo } & \multicolumn{2}{|c|}{ Once } \\
\hline & $M$ & D.T & M & D.T & M & D.T & M & D.T & M & D.T & M & D.T \\
\hline Antisocial & 2.56 & 4.86 & 4.15 & 4.70 & 4.77 & 4.85 & 6.11 & 5.57 & 7.87 & 5.20 & 6.92 & 6.18 \\
\hline Delictiva & 1.46 & 3.48 & 2.12 & 4.01 & 2.03 & 3.88 & 1.91 & 3.77 & 1.49 & 2.69 & 1.44 & 2.35 \\
\hline
\end{tabular}

Tabla 12

Grados de libertad, media cuadrática, Fy nivel de significancia de la conducta antisocial y delictiva de los adolescentes en función de la Escolaridad.

\begin{tabular}{lcccc}
\hline Escalas & Gl & $\begin{array}{c}\text { Media } \\
\text { cuadrática }\end{array}$ & F & Sig \\
\hline Antisocial & 5 & 550.092 & 20.739 & .000 \\
\hline Delictiva & 5 & 12.933 & 1.088 & .365 \\
\hline
\end{tabular}

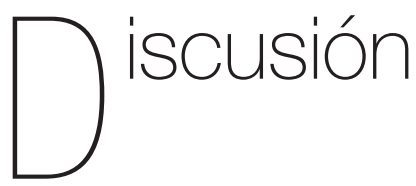

De acuerdo con los resultados, se evidencia que los adolescentes presentan conductas antisociales y delictivas, con mayor frecuencia antisociales frente a las delictivas. En cuanto a la presencia de las conductas antisociales son más reiterativas aquellas como el mal uso de palabras y/o groserías, aspectos relacionados al incumplimiento o llegada tarde al colegio, ingerir alimentos en lugares donde es prohibido, llamar a la puerta de alguien y salir corriendo, negarse a hacer las tareas, pelearse con otros, hacer trampa, tirar basura al suelo así haya una papelera cerca, y realizar bromas pesadas a otros; este tipo de conductas resultan realmente preocupantes en el desarrollo de la adolescencia, especialmente porque involucra el aspecto educativo como el hecho de no asistir a clase, ser expulsado y no tener continuidad en un colegio, el cual es relevante para la formación y el proceso de socialización del individuo. De acuerdo con Cabrera et al. (2012), es importante el control de las mismas porque a causa de estas conductas muchos de los adolescentes no realizan o culminan un proceso académico exitoso a causa de expulsiones, suspensiones y perdida de materias; y sumado a ello, se dan las escasas relaciones con el otro debido a que dentro de esa relación hayan víctimas, razón por la cual en la etapa adulta, muchos de ellos, se involucradan en problemas de alto riesgo psicosocial (Sanabria \& Uribe, 2009; 2010).

Con relación a las conductas delictivas que se presentan con mayor frecuencia en los adolescentes, pertenecer a una pandilla, es una acción que se ha ido configurando en el ambiente escolar como una forma de violencia visible (Sanabria \& Uribe, 2010) del que se desprenden otro tipo de conductas como armar desorden, peleas y genera disturbios, acciones que de acuerdo con Herrera y Morales (2005) ponen cada vez en alerta a la población mundial. En continuidad frente a la presencia de actos delictivos, se identificaron el mal gasto de dinero en juegos, entrar a un club prohibido o comprar bebidas prohibidas, llevar consigo un arma por si es necesario una pelea, forcejear o pelear 
con la policía, planear atentados, robar y forzar la entrada de un almacén. Estos tipos de datos muestran la importancia de realización de planes de acción que generen en el adolescente acciones prosociales y protectoras, frente a su vida y frente a quienes lo rodean, de tal manera que con esto se disminuyan los actos delictivos favoreciendo el desarrollo personal, social y económico del país (OMS, 2003).

En cuanto a la diferencia por género, se siguen presentando mayor conductas antisociales en hombres que en las mujeres, resultados que sustentan lo hallado en otras investigaciones (Gaeta \& Galvanovskis, 2011; Garaigordobil, 2005; Herrera \& Morales, 2005; Montero, 2014; Pérez et al., 2014; Sanabria \& Uribe, 2009; Uribe, 2005). De acuerdo con Gaeta y Galvanovskis (2011), las conductas antisociales de las mujeres están más relacionadas con aspectos como hacer trampa y llegar tarde al colegio mientras que en los hombres se presentan conductas con mayor contenido agresivo (Sanabria \& Uribe, 2009). Ante esto, puede decirse, que en ocasiones este tipo de conductas parecieran estar delimitadas por estereotipos sociales configurados frente al actuar entre el hombre y la mujer, aspectos que siguen delimitando las acciones de acuerdo al género y ante lo que es "socialmente es aceptado", pareciera que las conductas delictivas fueran "normalizadas" dentro de la configuración del comportamiento del hombre. Valdría la pena revisar a profundidad estas configuraciones sociales que se han creado frente a lo "bien visto o mal visto" desde la conducta antisocial y delictiva en los adolescentes de acuerdo al género.
Por otra parte, dentro de lo hallado se evidencia mayor índice de conductas antisociales de los adolescentes en la ciudad de Bogotá seguido de Armenia y Cali, y en cuanto a las conductas delictivas se presentan con mayor puntuación en la ciudad de Cali y Armenia. Estos aspectos que se relacionan de acuerdo a las cifras de violencia y guerra en el estudio nacional realizado por Restrepo y Aponte (2009), quienes señalaron que las ciudades de Cali, Cúcuta, Manizales y Pereira son las ciudades grandes de Colombia con mayor tasa de homicidios; así mismo, señalaron que Cali es la ciudad donde se presentaban más daños hacia las otras personas como las lesiones personales, en comparación con las otras ciudades; y frente al hurto a personas, ésta conducta delictiva con mayor frecuencia se presenta en la ciudad de Bogotá seguida de Cali y Manizales. Acorde a lo expuesto, es relevante mencionar que esto es coherente a lo referido por Pérez et al. (2014) en cuanto al aumento progresivo de las conductas delictivas con la edad y la repercusión y los riesgos que ello puede representar en la edad adulta para la persona (Giraldo et al., 2015). De acuerdo con lo mencionado, se sigue siendo indiferente ante las acciones de violencia que cada vez parecieran ser más arraigadas en ciertas zonas del país, lo cual sigue alimentando una sociedad violenta, que actúa ante este tipo de problemáticas con la imposición de duros castigos y acciones imperantes que a lo mejor pudo tener solución mucho antes. La cuestión fundamental radica, en que este tipo de conductas deben ser orientadas a la resolución desde diferentes escenarios en los que se tengan en cuenta los antecedentes y las condiciones sociales de la población. 
Por otra parte, se identificaron diferencias significativas en función del estrato socioeconómico y la escolaridad. En cuanto al estrato socioeconómico los adolescentes pertenecientes a estratos dos y tres presentan mayor puntuación en conductas antisociales, mientras que las conductas delictivas se presentan con mayor incidencia en los adolescentes de estratos cuatro y cinco. De acuerdo a lo manifiesto, los factores externos o el ambiente del exosistema (Sanabria \& Uribe, 2010) siguen siendo una variable importante e influyente para el desarrollo de las conductas delictivas y sobre todo para la predicción de las mismas (Giraldo et al., 2015; Peña, 2010). En este mismo sentido, la comunicación y el apoyo familiar son fundamentales en la medida que proporciona herramientas a los adolescentes para que aprendan a protegerse de situaciones y personas que pueden dañarlos, aspectos que contribuyen a mantener monitoreo y supervisión parental de una forma cómoda para el adolescente y en este mismo sentido, actúa como un factor de protección para los adolescentes que viven en ambientes de alto riesgo (Carrillo, Juárez, González-Forteza, Martínez, \& MedinaMora, 2016; Jiménez, Estévez, \& Murgui, 2014).

En este sentido, de acuerdo con la literatura y los resultados hallados, los comportamiento antisociales están influenciados por diferentes tipos de variables, entre ellas, predisposiciones biológicas, componentes ambientales, aspectos psicológicos como el tipo de personalidad (Human-Hendricks, \& Roman, 2014), la crianza familiar y el grupo de pares (Harris-McKoy \& Cui, 2013), los cuales han sido fundamentales a la hora de sustentar el comportamiento antisocial y/o delictivo que en este estudio, no se tuvo en cuenta a pesar de su relevancia, sin embargo, vale la pena mencionar que los estudios longitudinales (Harris-McKoy, \& Cui, 2013) y los meta-anlaisis (Human-Hendricks \& Roman, 2014) sustentan que en la etapa adolecente, el acompañamiento de los padres es fundamental para el desarrollo de la persona, los cuales a su vez configura el marco de acciones en los que se desenvuelve el joven. Aspectos que a la hora de dar explicaciones a los comportamientos de los adolescentes, resultan sustanciales a la hora de dar respuestas efectivas desde una perspectiva psicosocial.

Finalmente, en relación a la escolaridad, se identificó que las conductas antisociales se presentan mayormente en los grados de escolaridad superiores como noveno, décimo y once, con un grado de puntuación más alto para el grado décimo. Y en cuanto a las conductas delictivas las puntuaciones son más altas en los grados séptimo y octavo, respectivamente. Lo hallado puede relacionase con la variable de edad, mencionada reiterativamente en otros estudios, como aspecto relevante para la presencia de conductas antisociales y/o delictivas, ya que se ha identificado que este tipo de conductas son directamente proporcionales a la edad, cuando se empiezan a presentar desde inicios muy tempranos (Gaeta \& Galvanovskis, 2011; Giraldo et al., 2015; Sanabria \& Uribe, 2009). Estas marcadas diferencias en cuanto al grado, pueden verse influenciada por variables anteriormente mensionadas como el ambiente social y el tipo de crianza en los estudiantes. 


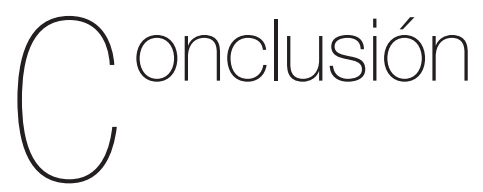

En el estudio se evidenciaron diferencias en las conductas antisociales y delictivas en cuanto a la ubicación geográfica, el sexo, la edad y el nivel de escolaridad. En este sentido, siguen siendo los varones quienes presentan con mayor frecuencia conductas antisociales y delictivas.

Estos resultados permiten vislumbrar implicaciones de carácter individual en los adolescentes, como bien son las dificultades en su desarrollo personal, a nivel psicológico la formación de personalidad con rasgos patológicos, problemas en el ámbito escolar debido al absentismo e insatisfacción en su desempeño académico; a nivel grupal, dificultad para relacionarse con otro grupo de pares, diferentes a sus amistades violentas que participa en actos agresivos; a nivel social el incremento de la delincuencia juvenil, el aumento de pandillas y la desintegración familiar.

Este tipo de conductas en los adolescentes, es un compromiso que compete a las alianzas en procesos formativos entre educadores, padres de familia, autoridades y profesionales de áreas afines, que de manera integral orienten soluciones viables a este tipo de problemática que se ha configurado en un problema a nivel mundial.

De acuerdo con estos hallazgos, es importante la implementación de programas de prevención en los que se tengan en cuenta todas las anteriores variables $y$ aquellas de tipo personal y familiar orientadas a disminuir los factores de riesgo en esta población. De igual forma, las relaciones parentales son fundamentales, en la medida que se estimule el apoyo y la comunicación en el ambiente familiar, orientando al adolescente a que se exprese libremente con sus padres, de manera que la supervisión parental se realice más fácilmente para ayudar a que estos menores tengan más herramientas que les ayuden a no reincidir y, no se involucren en actos antisociales.

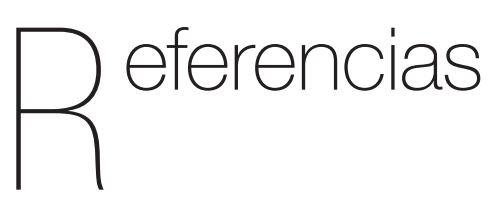

Arce, R., Fariña, F., \& Vázquez, M. J. (2011). Grado de competencia social y comportamientos antisociales, delictivos y no delictivos en adolescentes. Revista Latinoamericana de Psicología, 43(3), 473-486.

Cabrera, G., González, J., Vargas, L., \& Franco, L. (2012). Conducta antisocial y delictiva en adolescentes de un centro de reclusión en el Quindío, período 2008-2010. Psicogente, 15(27), 168-177.

Carrillo, L., Juárez, F., González-Forteza, C., Martínez, N. A., \& Medina-Mora, M. E. (2016). Relación entre supervisión parental y conducta antisocial en menores infractores del Estado de Morelos. Salud Mental, 39(1), 11-17. doi:10.17711/ SM.0185-3325.2015.063

Gaeta, M., \& Galvanovskis, A. (2011). Propensión a conductas antisociales y delictivas en adolescentes mexicanos. Psicología Iberoamericana, 19(2), 47-54. 
Garaigordobil, M. (2005). Conducta antisocial durante la adolescencia: Correlatos socioemocionales, predictores y diferencias de género. Psicología Conductual, 13(2), 197 215.

Giraldo, H., Ocampo, J. F., Tuta, F. A., Gallego, M. C., \& López, Y. (2015). ¿Por qué las personas ingresan a la delincuencia organizada? Análisis del fenómeno en Santiago de Cali. Revista Criminalidad, 57(1), 103-119.

Harris-McKoy, D., \& Cui, M. (2013). Parental Control, Adolescent Delinquency, and Young Adult Criminal Behavior. Journal of Child \& Family Studies, 22(6), 836-843. doi:10.1007/s10826-012-9641-x

Hernández, R., Fernández, C., \& Baptista, P. (2010). Metodología de la investigación (5aed). Santa Fé de Bogota: MacGraw-Hill

Herrera, D., \& Morales, H. (2005). Comportamiento antisocial durante la adolescencia: Teoría, investigación y programas de prevención. Revista de Psicología de la PUCP, 23(2), 201-247.

Hoeve, M., Semon-Dubas, J., Eichelsheim, V. I., van der Laan, P. H., Smeenk, W., \& Gerris, J. R. M. (2009). The relationship between parenting and delinquency: A meta-analysis. Journal of Abnormal Child Psychology, 37, 749-775.

Human-Hendricks, A. R., \& Roman, N. V. (2014). What is the link between antisocial behavior of adolescents and parenting: Asystematic review of parental practices to manage antisocial behavior. Journal of Communications Research, 6(4), 439-464.

Lüdke, F., Mendes, S., Bizarro, L., \& Dalbosco, D. (2012). Drug use and antisocial behavior among adolescents attending public schools in Brazil. Trends Psychiatry Psychother, 34(2), 80-86.

Jiménez, T. I., Estévez, E., \& Murgui, S. (2014). Ambiente comunitario y actitud hacia la autoridad: Relaciones con la calidad de las relaciones familiares y con la agresión hacia los iguales en adolescentes. Anales de Psicología, 30(3), 1086-1095. doi:10.6018/ analesps.30.3.160041

Jordá S. C., \& Besteiro Y. I. (2013). La psicopatía en menores infractores: Retos para su investigación e intervención. Revista Criminalidad, 55(3), 265-278.

Kazdin, A. (1995). Conduct disorder in childhood and adolescence (2a. edition). Newbury Park, CA: Sage.

Kazdin, A. E., \& Buela-Casal, G. (1996). Conducta antisocial evaluación, tratamiento y prevención en la infancia y adolescencia. Madrid: Ediciones Pirámide.

López, C., \& López, J. R. (2003). Rasgos de personalidad y conducta antisocial y delictiva. Psicopatología Clínica, Legal y Forense, 3, 5-19.

Montero H., T. (2014). La criminalidad juvenil en España (2007-2012). Revista Criminalidad, 56(2), 247-261.

Morán, C., Carmona, J. A., \& Fínez, M. J. (2016). Tipos de personalidad, agresión y conducta antisocial en adolescentes. Psychology, Society \& Education, 8(1), 65-80.

Morales, H. (2008). Factores asociados y trayectorias del desarrollo del comportamiento antisocial durante la adolescencia: Implicancias para la 
prevención de la violencia juvenil en América Latina. Revista Interamericana de Psicología, 42(1), 129-142.

Organización Mundial de la Salud. (OMS, 2003). Informe mundial sobre la violencia y la salud. Washington, DC. Recuperado de http://apps.who.int/iris/ bitstream/10665/112670/1/9275315884_ spa.pdf

Pelegrín, A., \& Garcés de Los Fayos, E. (2009). Análisis de las variables que influyen en la adaptación y socialización: El comportamiento agresivo en la adolescencia. Ansiedad y Estrés, 15(2/3), 131-150.

Peña, M. (2010). Conducta antisocial en adolescentes: Factores de riesgo y de protección. (Tesis doctoral). Universidad de Granada, Granada.

Pérez, M., Molero, M., Gázquez, J., \& Abad, T. (2014). Análisis de las conductas antisociales-delictivas en la etapa de educación secundaria: edad, género y perfil del alumno en la convivencia. Proceedings of International Congress of Clinical Psychology, 6(8), 35-41.
Restrepo, J., \& Aponte, D. (2009). Guerra y violencias en Colombia: Herramientas e interpretaciones (1a ed). Bogotá: Editorial Pontificia Universidad Javeriana.

Sanabria, A. M., \& Uribe, A. (2010). Factores psicosociales de riesgo asociados a conductas problemáticas en jóvenes infractores y no infractores. Diversitas, 6(2), 257-274.7.

Sanabria, A., \& Uribe, A. (2009). Conductas antisociales y delictivas en adolescentes infractores y no infractores. Pensamiento Psicológico, 6(13), 203-221.

Schroeder, R. D., Bulanda, R. E., Giordana, P. C., \& Cernkovich, S. A. (2010). Parenting and adult criminality: An examination of direct and indirect effects by race. Journal of adolescent Research, 25, 64-98.

Uribe, A. F. (2005). Evaluación de factores psicosociales de riesgo para la infección por el VIH/sida en adolescentes colombianos (Tesis doctoral). Universidad deGranada, Granada. 